\title{
A Journey Between Two Curves
}

\author{
Sergey A. CHERKIS ${ }^{\dagger^{1} \dagger^{2}}$ \\ $\dagger^{1}$ School of Mathematics, Trinity College Dublin, Ireland \\ E-mail: cherkis@maths.tcd.ie \\ $\dagger^{2}$ Hamilton Mathematics Institute, TCD, Dublin, Ireland
}

Received October 31, 2006, in final form February 25, 2007; Published online March 11, 2007

Original article is available at http://www.emis.de/journals/SIGMA/2007/043/

\begin{abstract}
A typical solution of an integrable system is described in terms of a holomorphic curve and a line bundle over it. The curve provides the action variables while the time evolution is a linear flow on the curve's Jacobian. Even though the system of Nahm equations is closely related to the Hitchin system, the curves appearing in these two cases have very different nature. The former can be described in terms of some classical scattering problem while the latter provides a solution to some Seiberg-Witten gauge theory. This note identifies the setup in which one can formulate the question of relating the two curves.
\end{abstract}

Key words: Hitchin system; Nahm equations; monopoles; Seiberg-Witten theory

2000 Mathematics Subject Classification: 53C28; 53C80; 70H06; 81T30

\section{Introduction}

The Nahm Equations [1] and the Hitchin Equations [2, 3] are two integrable systems of equations that play increasingly important role in string theory and gauge theory literature. Solutions of Seiberg-Witten gauge theories in four [4, 5] and in three dimensions [6], dynamics of a five brane on a holomorphic curve [7, 8], D-brane dynamics [9, 10], aspects of the Generalized Geometry [12], physics version of Langlands duality [13], domain walls [14], topological YangMills and nonlinear Schrödinger [15], to name a few, can be described in their terms.

The two systems are closely related. In fact, one can think of the Nahm equations as a limit of those of Hitchin. As integrable systems each can be solved in terms of a holomorphic curve and a point or a linear flow on its Jacobian. Yet, these two solutions are tantalizingly different.

Each system can be mapped to a different problem by the Nahm transform. The Nahm transform on a four-torus is presented beautifully in [16]; a general review of the Nahm transform can be found in [17]. A solution to the Nahm equations is mapped to a monopole [18, 19], i.e. solutions of the Bogomolny equation. In turn, a solution to the Hitchin equations defined on a torus is mapped to a doubly-periodic instanton [20]. A reduction of the instanton equation leads to the Bogomolny equation. Since instanton and Bogomolny equations are, naturally, also integrable, one can find the respective curves describing their solutions. Yet again, the two curves differ drastically from each other.

In the context of monopoles and the Hitchin system respectively both curves $\mathbf{S}$ and $\boldsymbol{\Sigma}$ were introduced by Hitchin and both are often referred to as Hitchin Spectral Curves. To distinguish these curves we shall refer to $\mathbf{S}$ as the Twistor Curve and to $\boldsymbol{\Sigma}$ as the Higgs Curve. The two are of the very different nature, nevertheless, they are closely related. As we hope to demonstrate here, this relation is deeper than originating from the same mind.

${ }^{\star}$ This paper is a contribution to the Proceedings of the Workshop on Geometric Aspects of Integrable Systems (July 17-19, 2006, University of Coimbra, Portugal). The full collection is available at http://www.emis.de/journals/SIGMA/Coimbra2006.html 


\section{The physics of the Higgs Curve $\Sigma$ and the Twistor Curve S}

Our motivation for posing the problem comes from the deep significance of both curves in the study of gauge theories with eight supercharges. A solution to this problem would also provide a glimpse at the nonperturbative dynamics of the M theory five-brane.

\section{$2.1 \quad \Sigma$ in Seiberg-Witten theory}

The solution of the $\mathcal{N}=2$ gauge theory found by Seiberg and Witten [21, 22] is formulated in terms of an auxiliary curve. This is a holomorphic curve corresponding to a choice of a vacuum of the theory. It is referred to as the Seiberg-Witten curve. As demonstrated in [4], Seiberg-Witten curve is exactly the Higgs Curve $\boldsymbol{\Sigma}$, as outlined in Section 4.1 below.

M theory significance of the Seiberg-Witten curve was uncovered in $[7,11,8]$. In [8] the curve $\boldsymbol{\Sigma}$ emerges as a curve on which the M theory five-brane is wrapped. The five brane dynamics is equivalent to that of a type IIA string theory brane configuration of ChalmersHanany-Witten [9] and [10]. In turn, the low energy dynamics of this brane configuration is described by a Seiberg-Witten gauge theory. Thus, what appeared to be an auxiliary curve of Seiberg and Witten acquired new meaning as the shape of the M theory five-brane.

Another path to this $M$ theory interpretation was discovered earlier in [7, 11]. In this Geometric Engineering scenario the Seiberg-Witten gauge theory emerges from the compactification of the IIA string theory on a Calabi-Yau space with a prescribed singular structure along a curve in it. After local T-duality this Calabi-Yau space is mapped to a five-brane wrapped on a SeibergWitten curve.

\subsection{Three-dimensional Gauge theories and S}

The gauge theory relevant to this section is $\mathcal{N}=4$ supersymmetric gauge theory in three dimensions. The case of pure $\mathcal{N}=4$ Yang-Mills was considered in [6], where the geometry of its moduli space of vacua is identified with the Atiyah-Hitchin moduli space [23]. Chalmers, Hanany, and Witten in [9] and [10] have identified the brane configuration that allows one to associate the above quantum field theory problem with a problem of the classical dynamics of monopoles. It also makes the relation to the corresponding Nahm data transparent. It even allows one to re-derive the Nahm transform from the string theory [24]. Using these ideas one can identify the exact monopole problem solving three-dimensional $\mathcal{N}=4$ QCD [25]. One can also derive the Kähler potential on the moduli space [26]. The auxiliary curve $\mathbf{S}$ plays central role in this derivation. Moreover, using the information encoded in $\mathbf{S}$ (and a bundle $\mathcal{L} \rightarrow \mathbf{S}$ ) one can obtain the explicit metric on the moduli space [27]. From the point of view of the original quantum gauge theory this metric contains all of the nonperturbative corrections.

The physical significance of the twistor curve $\mathbf{S}$ is much less clear. It is also much harder to find it explicitly, since one has to satisfy the transcendental constraint, as stated in Section 3.3. However, the curve $\mathbf{S}$ contains complete information about the moduli space, as well as about the solutions of the associated monopole and Nahm problems themselves.

\section{$2.3 \Sigma$ in Seiberg-Witten theories on $\mathbb{R}^{3} \times S^{1}$}

Supersymmetric $\mathcal{N}=2$ gauge theories on a space with one compact direction considered in [6] are rather revealing, since they interpolate between the four- and three-dimensional theories. From the geometric point of view, they also have much more interesting moduli spaces of vacua.

Applying the techniques of the previous section one can identify the Chalmers-HananyWitten type brane configuration corresponding to the quantum gauge theory. From this one can read off the corresponding Hitchin system [28] and via T-duality obtain the periodic monopole 
description [29]. Both lead to the curve $\boldsymbol{\Sigma}$ which is the Seiberg-Witten curve of the quantum gauge theory problem.

Most importantly, the curve $\boldsymbol{\Sigma}$ is relatively easy to find explicitly. It contains a wealth of information about the Seiberg-Witten gauge theory on $\mathbb{R}^{3} \times S^{1}$; however, it is far from sufficient to know $\boldsymbol{\Sigma}$ in order to find the effective low energy theory in this case. Physically, the main difference between the quantum field theory on $\mathbb{R}^{4}$ and a theory on $\mathbb{R}^{3} \times S^{1}$ is that in addition to the instanton corrections the latter also has monopole corrections, since in that case a selfdual configuration independent of $S^{1}$ has finite action. The Seiberg-Witten curve $\boldsymbol{\Sigma}$ accounts for all perturbative and instanton corrections to the effective theory, but not the monopole contributions.

Let us comment on the M theory significance of the problem we suggest. As explained in [8], in the extreme infra-red the effective field theory of a Seiberg-Witten theory is given by the low energy dynamics of an M theory five-brane wrapped on a curve $\boldsymbol{\Sigma}$. The reduction of the $\mathrm{M}$ theory action produces the Seiberg-Witten solution [30]. However, if the gauge theory is considered on $\mathbb{R}^{3} \times S^{1}$ the five brane dynamics receives membrane instanton corrections. In this case the five-brane wraps $\boldsymbol{\Sigma} \times S^{1}$ and for some one-cycle $\Gamma \subset \boldsymbol{\Sigma}$ an instanton membrane of finite volume can have $\Gamma \times S^{1}$ as its boundary. Such a membrane instanton produces corrections to the five-brane dynamics. At low energies, these can be interpreted as monopole corrections in the corresponding gauge theory.

The main purpose of this paper is to highlight the relation between $\mathbf{S}$ and $\boldsymbol{\Sigma}$. SeibergWitten theories on a space with one compact direction provide a perfect framework to pose this question. In physics terms, a vacuum of such a theory defines a curve $\boldsymbol{\Sigma}$, it also defines what

we call a Twistor Curve $\mathbf{S}_{p}$. Thus, we expect these two to be related. Moreover, we expect the family of $\mathbf{S}_{p}$ curves to contain the complete information about the moduli space of vacua.

\section{Two Descriptions of the Twistor Curve S: monopoles and Nahm equations}

There is a well established relation, discovered by Werner Nahm in [1, 18, 19], between solutions $(A, \Phi)$ of the Bogomolny equation

$$
\star F=D \Phi
$$

and solutions of the system of the Nahm equations

$$
\begin{aligned}
\frac{d}{d s} T_{1}(s)+i\left[T_{0}(s), T_{1}(s)\right] & =-i\left[T_{2}(s), T_{3}(s)\right], \\
\frac{d}{d s} T_{2}(s)+i\left[T_{0}(s), T_{2}(s)\right] & =-i\left[T_{3}(s), T_{1}(s)\right], \\
\frac{d}{d s} T_{3}(s)+i\left[T_{0}(s), T_{3}(s)\right] & =-i\left[T_{1}(s), T_{2}(s)\right] .
\end{aligned}
$$

This relation provides a one-to-one map between solutions of equation (1) and solutions of the system of equations (2). It is a nonlinear version of the Fourier transform called the Nahm transform.

\subsection{The monopole Spectral Curve}

Before we begin, let us recall the notion of the minitwistor space $\mathbf{T}$, which, in the case of a flat three-dimensional space, is the space of lines in $\mathbb{R}^{3}$. A line in $\mathbb{R}^{3}$ can be written in the form $\vec{x}=t \vec{n}+\vec{v}$, where $\vec{n}$ is a unit direction vector and $\vec{v}$ is the displacement vector orthogonal to $\vec{n}$. 
Each oriented line is uniquely specified by the pair $(\vec{n}, \vec{v})$ with $|\vec{n}|=1$ and $\vec{v} \cdot \vec{n}=0$. Thus the space of lines in $\mathbb{R}^{3}$ forms the tangent space to a two-sphere, where $\vec{n}$ specifies the point on the two-sphere and $\vec{v}$ specifies a point in the tangent space at it. Identifying the two-sphere with the Riemann sphere with a coordinate $\zeta$, we give the minitwistor space $\mathbf{T}$ a natural complex structure. Thus $\mathbf{T}=T \mathbb{P}^{1}$ and locally we introduce coordinate $\eta$ in the fiber, so that $\eta \frac{\partial}{\partial \zeta} \in T \mathbb{P}^{1}$.

As proposed by Nigel Hitchin in [31], to every solution $(A, \Phi)$ of the Bogomolny equation one can associate a scattering problem. For each line consider a differential equation

$$
\left(D_{\vec{n}}+\Phi\right) \Psi=0
$$

where $D_{\vec{n}}$ is the covariant derivative along the line and $\Psi$ is a section of the restriction of the bundle $E$ to this line. For some lines this problem has a bound state, i.e. a square integrable solution $\Psi$. We call such a line spectral. Given a monopole solution of charge $N$ for any unit vector $\vec{n}$ generically there will be exactly $N$ spectral lines along $\vec{n}$. Since every line in $\mathbb{R}^{3}$ corresponds to a point in $\mathbf{T}$, the set of points corresponding to spectral lines forms a curve $\mathbf{S}$ in $\mathbf{T}$. Thanks to the fact that $(A, \Phi)$ satisfied Bogomolny equation, this curve $\mathbf{S}$ is holomorphic. Moreover, since a point on this curve corresponds to a line in $\mathbb{R}^{3}$ with a nontrivial square integrable solution $\Psi$ of equation (3), we obtain a line bundle $\mathcal{L}$ over $\mathbf{S}$. See [31] and [32] for details.

So far we have outlined a map which for every monopole $(A, \Phi)$ produces a pair $(\mathbf{S}, \mathcal{L})$ of the curve and the bundle. These satisfy certain conditions formulated below in Section 3.3. So long as these conditions are satisfied the map is one-to-one [31,23]. For a monopole of charge $N$ the curve $\mathbf{S} \subset T \mathbb{P}^{1}$ is an $N$ fold cover of the base $\mathbb{P}^{1}$. In other words, knowing $\mathbf{S} \subset T \mathbb{P}^{1}$ and $\mathcal{L} \rightarrow \mathbf{S}$ we can, in principle, reconstruct a unique monopole solution (up to a gauge transformation).

\subsection{Nahm Spectral Curve}

The Nahm transform is a one-to-one map, mapping a monopole of charge $N$ to a solution of the Nahm equations where $T_{j}(s)$ are functions of the variable $s$ with $N \times N$ Hermitian matrix values. Now we briefly outline how the same curve $\mathbf{S}$ and the line bundle $\mathcal{L}$ emerge from the corresponding Nahm data $\left(T_{0}(s), T_{1}(s), T_{2}(s), T_{3}(s)\right)$.

Introducing an auxiliary parameter $\zeta$ define

$$
\begin{aligned}
& A=i T_{0}+T_{3}+\zeta\left(T_{1}+i T_{2}\right), \\
& L=-T_{1}+i T_{2}+2 i \zeta T_{3}+\zeta^{2}\left(T_{1}+i T_{2}\right),
\end{aligned}
$$

then the system of the Nahm equations (2) can be written as

$$
\frac{d}{d s} L+[A, L]=0
$$

The above equation implies that the eigenvalues of $L$ are independent of $s$, thus any solution of the Nahm equations defines a spectral curve $\mathbf{S} \subset \operatorname{Tot} \mathcal{O}(2)=T \mathbb{P}^{1}$ given by

$$
\mathbf{S}: \operatorname{det}(L-\eta)=0
$$

Since the curve is the curve of eigenvalues the corresponding eigenspaces form a line bundle $\mathcal{L}$. These are exactly the same as the curve and the bundle of the corresponding monopole constructed in the previous section.

\subsection{The Twistor Curve constraints}

The constraint that every twistor curve satisfies is formulated in terms of a line bundle $L$ over the minitwistor space $\mathbf{T}$. In terms of the coordinates $(\zeta, \eta)$ introduced above the line bundle 
$L^{x}(m)$ is defined by its transition function $\zeta^{-m} e^{-x \eta / \zeta}$. There is a natural restriction of this bundle $\left.L\right|_{S}$ to any curve $S$. In case of a regular $S U(2)$ monopole of charge $K$ the "vanishing theorem" of [32] states that the twistor curve satisfies the following conditions:

$$
\begin{array}{ll}
\left.L^{x}(k-2)\right|_{\mathbf{S}} & \text { is nontrivial for } 0<x<2, \\
\left.L^{2}\right|_{\mathbf{S}} & \text { is trivial. }
\end{array}
$$

\section{Two descriptions of the Spectral Curve $\Sigma$ : the Hitchin system and instantons}

The Hitchin system of equations is written for a Hermitian gauge field $A=A_{1} d x^{1}+A_{2} d x^{2}$ and a pair of Higgs fields $\Phi_{1}$ and $\Phi_{2}$ on some Riemann surface $X$. The corresponding connection is $D_{j}=\partial_{j}+i A_{j}$ and the equations are

$$
\begin{aligned}
& {\left[D_{1}, D_{2}\right]=-\left[\Phi_{1}, \Phi_{2}\right],} \\
& {\left[D_{1}, \Phi_{1}\right]=-\left[D_{2}, \Phi_{2}\right],} \\
& {\left[D_{1}, \Phi_{2}\right]=\left[D_{2}, \Phi_{1}\right] .}
\end{aligned}
$$

The Nahm transform maps any solutions of the Hitchin system on a torus $T^{2}$ to a doubly-periodic instanton [20,33].

\subsection{The Higgs Curve $\Sigma$ from the Hitchin system}

The Hitchin system can be written in the following form

$$
\begin{aligned}
& {[D, \bar{D}]=-\left[\Phi, \Phi^{\dagger}\right],} \\
& {[\bar{D}, \Phi]=0,}
\end{aligned}
$$

if we put $D=D_{1}-i D_{2}, \Phi=\Phi_{1}-i \Phi_{2}$, so that $\bar{D}=D_{1}+i D_{2}$ and $\Phi^{\dagger}=\Phi_{1}+i \Phi_{2}$. The second one of these, equations (6), implies that the curve $\boldsymbol{\Sigma} \subset T^{*} X$ defined by

$$
\boldsymbol{\Sigma}: \operatorname{det}(\Phi-w)=0,
$$

is holomorphic. We shall refer to this curve as the Higgs Curve or the Brane Curve. Since $\boldsymbol{\Sigma}$ is a curve of eigenvalues it comes with the line bundle $\mathcal{N} \rightarrow \boldsymbol{\Sigma}$. Each fiber of $\mathcal{N}$ is the corresponding eigenspace.

Thanks to the Donaldson's theorem [34] extended to this case $(\boldsymbol{\Sigma}, \mathcal{N})$ define the solution of the Hitchin system uniquely (up to a gauge transformation).

\subsection{The Higgs Curve from doubly-periodic instantons}

A doubly-periodic instanton is a self-dual connection on $\mathbb{R}^{2} \times T^{2}$. Let us introduce complex coordinates $z \in \mathbb{C} \simeq \mathbb{R}^{2}$ and $w \in T^{2} \simeq \mathbb{C} /(\mathbb{Z} \times \mathbb{Z})$. Instanton equations can be written as

$$
\begin{aligned}
& {\left[D_{z}, D_{w}\right]=0} \\
& {\left[D_{z}, \bar{D}_{z}\right]=\left[D_{w}, \bar{D}_{w}\right] .}
\end{aligned}
$$

As demonstrated in [33], following the ideas of [35, 36], a spectral curve is associated to any solution of these equations in the following manner. For any point $z \in \mathbb{C}$ we can consider monodromies of a flat connection on the torus $T_{z}^{2}$ with the holomorphic combination equal to $D_{w}$ of the instanton. If the instanton bundle is of rank $K$, then the eigenvalues of these 
monodromies correspond to $K$ points in the dual torus $\check{T}^{2}$. Thus the set of all these points for all values of $z$ defines a curve $\boldsymbol{\Sigma}$ which is a $K$ fold covering of $\mathbb{R}^{2}$. Thanks to the fact that the initial connection satisfied the first equation in (7), this curve $\boldsymbol{\Sigma}$ is holomorphic.

It is no coincidence that the curves of Section 4.2 and Section 4.1 are both denoted by $\boldsymbol{\Sigma}$. As proved in [33], if a doubly-periodic instanton (7) is related via the Nahm transform to a solution of the Hitchin system (6) the corresponding curves coincide.

\section{$5 \quad$ A bridge between $\mathrm{S}$ and $\Sigma$ : periodic monopoles}

One might consider various limits in order to relate the two curves. For example, in a certain limit the Hitchin system degenerates to a system of the Nahm equations and doubly-periodic instanton become a monopole. In this section, however, we explore the case where the curves of both kinds appear in the same problem.

Let us consider periodic monopoles [29], i.e. solutions of Bogomolny equation (1) on $\mathbb{R}^{2} \times S^{1}$. What are the corresponding curves in this situation?

\subsection{The Higgs Curve $\Sigma$ for periodic monopoles}

Identifying the $\mathbb{R}^{2}$ of $\mathbb{R}^{2} \times S^{1}$ with a complex plane $\mathbb{C}$ with a coordinate $z$ we consider the monodromy $W(z)$ of the modified connection $D+\Phi$ around the circle $S_{z}^{1}$. In other words, if $w(z, 0)=1$ and

$$
\left(\frac{\partial}{\partial \varphi}+i A_{\varphi}+\Phi\right) w(z, \phi)=0
$$

then $W(z)=w(z, R)$. Here $R$ is the period of the coordinate $\varphi$ along the $S^{1}$ at $z$. As argued in [29], the curve defined by the following equation is holomorphic.

$$
\boldsymbol{\Sigma}_{p}: \operatorname{det}(W(z)-t)=0 .
$$

Since $t$ is an eigenvalue of a monodromy matrix it is nonzero, thus $t \in \mathbb{C}^{*}$ and $z \in \mathbb{C}$, and $\boldsymbol{\Sigma}_{p} \subset \mathbb{C} \times \mathbb{C}^{*}$.

\subsection{The Twistor Curve $\mathrm{S}$ for periodic monopoles}

In order to define the scattering problem in this case, we start with describing the minitwistor space of $\mathbb{R}^{2} \times S^{1}$. We can view $\mathbb{R}^{2} \times S^{1}$ as the quotient of $\mathbb{R}^{3}$ with respect to integer shifts by a vector $\vec{R} \in \mathbb{R}^{3}$, with $R=|\vec{R}|$. A point in $\mathbb{R}^{3}$ determines a section of $T \mathbb{P}^{1}$ in the following way. The set of all oriented lines passing through $\vec{R}$ forms a sphere. Each of these lines corresponds to a unique point in $\mathbf{T}=T \mathbb{P}^{1}$, thus $\vec{R}$ corresponds to a section $p_{\vec{R}}$ of the tangent bundle $T \mathbb{P}^{1}$. Let $p(\zeta)=-x_{1}+i x_{2}+2 x_{3} \zeta+\left(x_{1}+i x_{2}\right) \zeta^{2}$. In terms of the local coordinates $(\zeta, \eta)$, that we defined above, $p_{\vec{R}}: \eta=p(\zeta)$.

Shifting $\mathbb{R}^{3}$ by $\vec{R}$ maps a point $\left(\zeta_{0}, \eta_{0}\right)$ of $T \mathbb{P}^{1}$ to $\left(\zeta_{0}, \eta_{0}+p\left(\zeta_{0}\right)\right)$. Thus the minitwistor space $\mathbf{T}_{p}$ of $\mathbb{R}^{2} \times S^{1}$ is $T \mathbb{P}^{1} / \mathbb{Z} p_{\vec{R}}$. Here the subscript $p$ in $\mathbf{T}_{p}$ stands for 'periodic'. Note that the the fiber above $\zeta$ such that $p(\zeta) \neq 0$ is $\mathbb{C} / \mathbb{Z} \simeq \mathbb{C}^{*}$, while the fibers above $\zeta_{1}$ and $\zeta_{2}$ are $\mathbb{C}_{\zeta_{1}}=\mathbb{C}$ and $\mathbb{C}_{\zeta_{2}}=\mathbb{C}$. Now we have the minitwistor space $\mathbf{T}_{p}$ for $\mathbb{R}^{2} \times S^{1}$. Alas, the space $\mathbf{T}_{p}$ is not Hausdorff. In order to avoid this problem for now, let us consider the compliment of the two fibers above the roots $\zeta_{1}$ and $\zeta_{2}$ of $p(\zeta)$. We shall denote it by $\mathbf{T}_{p}^{\mathrm{Reg}}$. In other words $\mathbf{T}_{p}^{\mathrm{Reg}}=\mathbf{T} \backslash\left(\mathbb{C}_{\zeta_{1}} \cup \mathbb{C}_{\zeta_{2}}\right)$. Without loss of generality we can choose the vector $\vec{R}$ to be along the third axis and work away from $\zeta=0$ and $\zeta=\infty$.

From the point of view of the geodesics on $\mathbb{R}^{2} \times S^{1}$ one can see why the roots of $p(\zeta)$ are so special. A generic geodesic is infinitely long and projects to a line in $\mathbb{R}^{2}$. There is a set of 
geodesics, however, that are circles each of which projects to a point on $\mathbb{R}^{2}$, namely, these are the geodesics directed along $\vec{R}$. Clearly, for any two of the circular geodesics we can find a long geodesic that is arbitrarily close to both. Excluding the circular geodesics for now, we avoid this problem.

For any long geodesic we can again consider the scattering problem of Hitchin. A geodesic is called spectral if, as in the case of monopoles, it has a square integrable solution $\Psi$ of

$$
\left(D_{\vec{n}}+\Phi\right) \Psi=0 .
$$

The set of spectral geodesics forms a curve $\mathbf{S}_{p} \subset \mathbf{T}_{p}^{\mathrm{Reg}}$. This cure is holomorphic, which is a consequence of the Bogomolny equation.

\section{The Hitchin system}

The Nahm transform of a periodic monopole is a Hitchin system on $\mathbb{R} \times S^{1}$. Here we describe the curves $\boldsymbol{\Sigma}_{p}$ and $\mathbf{S}_{p}$ in terms of this Hitchin data $(A, \Phi)$.

The Higgs curve $\boldsymbol{\Sigma}_{p}$ of the eigenvalues of $\Phi_{1}-i \Phi_{2}$ is defined in the same way as for the general Hitchin system of Section 4.1. In our case the Hitchin data is defined on $\mathbb{R} \times S^{1} \simeq \mathbb{C}^{*}$ and thus the resulting curve lies in $T^{*} \mathbb{C}^{*}=\mathbb{C} \times \mathbb{C}^{*}$.

In order to define the Twistor Curve $\mathbf{S}_{p}$ let us follow [37] and introduce the so-called $\lambda$-connection

$$
D_{\zeta}=D+\zeta \Phi, \quad \bar{D}_{\zeta}=\bar{D}+\frac{1}{\zeta} \Phi^{\dagger}
$$

where the auxiliary parameter $\zeta \in \mathbb{C}^{*}$. Then the Hitchin equations (5) are equivalent to flatness of this connection: $\left[D_{\zeta}, \bar{D}_{\zeta}\right]=0$ for all values of $\zeta \in \mathbb{C}^{*}$. The monodromy of a flat connection on $\mathbb{R} \times S^{1}$ along a closed path winding once around the $S^{1}$ has eigenvalues independent of the choice of the path. Thus, for any nonzero $\zeta$ we obtain a set of points in $\mathbb{C}^{*}$. This defines the curve $\mathbf{S}_{p} \subset \mathbf{T}_{p}^{\mathrm{Reg}}$.

Clearly the curve $\mathbf{S}_{p}$ and the bundle $\mathcal{L}$ thus obtained satisfy some nontrivial conditions. In particular, in the limit of the zero radius of the $S^{1}$ factor of the $\mathbb{R} \times S^{1}$, the Hitchin system degenerates into the system of the Nahm equations. In this limit $\mathbf{S}_{p}$ becomes $\mathbf{S}$ of Section 3.2. Thus whatever the conditions the curve $\mathbf{S}_{p}$ has to satisfy should degenerate to the constraint of equation (4).

\section{The question}

For a generic periodic monopole, we expect the same theorems to hold as in the case of a monopole in $\mathbb{R}^{3}$ and in the case of doubly-periodic instantons. Namely, each pair $\left(\boldsymbol{\Sigma}_{p}, \mathcal{N}\right)$ of the curve $\boldsymbol{\Sigma}_{p}$ and a line bundle $\mathcal{N}$ is in one-to-one correspondence with a periodic monopole. On the other hand, each pair $\left(\mathbf{S}_{p}, \mathcal{L}\right)$ satisfying certain conditions is also in one-to-one correspondence with a periodic monopole. Thus there should be a one-to-one map mapping a pair $\left(\boldsymbol{\Sigma}_{p}, \mathcal{N}\right)$ to the pair $\left(\mathbf{S}_{p}, \mathcal{L}\right)$.

Since the Nahm transform relates periodic monopoles with the solutions of the Hitchin system on $\mathbb{R} \times S^{1}[28,29]$, periodic monopoles in the above argument can be substituted with that Hitchin system.

Now we are in a position to formulate the question: what is the explicit map relating the Higgs curve $\boldsymbol{\Sigma}$ to the Twistor Curve $\mathbf{S}$ ? 


\section{Appendix. Speculations}

Let us come back to the minitwistor space $\mathbf{T}_{p}$. The curve $\mathbf{S}_{p}$ was defined on the compliment of the 'problematic' fibers $\mathbb{C}_{\zeta=0}$ and $\mathbb{C}_{\zeta=\infty}$. The fiber $\mathbb{C}_{\zeta=0}$ can be identified with the $\mathbb{R}^{2}$ factor of the space $\mathbb{R}^{2} \times S^{1}$ on which the periodic monopole is defined. (The fiber $\mathbb{C}_{\zeta=\infty}$ is identified with the same $\mathbb{R}^{2}$ factor but with the opposite orientation.) Each point in this fiber corresponds to a closed geodesic $S^{1}$. It is exactly these geodesics that are used to define the Higgs Curve $\boldsymbol{\Sigma}_{p}$. Moreover, one cannot fail to notice that the operator in equation (8) is exactly the same as in the scattering problem equation (9). (Note, however, that $\Psi$ in equation (9) is a section, while $w$ in equation (8) is a parallel transport operator.) Thus, in some sense, the curve $\boldsymbol{\Sigma}_{p}$ (or rather its zeros with $t=0$ ) is a limit of $\mathbf{S}_{p}$ at the special fiber at $\zeta=0$ (and $\zeta=\infty$ ).

These is a potentially important difference between the role the curves $\boldsymbol{\Sigma}$ and $\mathbf{S}_{p}$ play in the integrable system. A particular doubly-periodic monopole solution (or a corresponding solution to the Hitchin system) is determined in terms of a curve $\boldsymbol{\Sigma}$ and a point in its Jacobian $\operatorname{Jac}(\boldsymbol{\Sigma})$, while in terms of the curve $\mathbf{S}_{p}$ such solution is determined by $\mathbf{S}_{p}$ and a linear flow on its Jacobian $\operatorname{Jac}\left(\mathbf{S}_{p}\right)$. These two cases correspond to a two different views on the monopole (or Hitchin equations). In the earlier case the solution is viewed as a point in the configuration space of an integrable system. In the latter case, the same solution is interpreted as an evolution trajectory of an integrable system.

\section{Acknowledgments}

We thank Pierre Deligne, Tamas Hausel, Nigel Hitchin, Anton Kapustin, Lionel Mason, Tony Pantev, Emma Previato, Samson Shatashvili, and Edward Witten for useful discussions.

This work is supported by the Science Foundation Ireland Grant No. 06/RFP/MAT050 and by the European Commision FP6 program MRTN-CT-2004-005104.

\section{References}

[1] Nahm W., A simple formalism for the BPS monopole, Phys. Lett. B 90 (1980), 413-414.

[2] Hitchin N., The self-duality equations on a Riemann surface, Proc. London Math. Soc. 55 (1987), 59-126.

[3] Hitchin N., Stable bundles and integrable systems, Duke Math. J. 54 (1987), 91-114.

[4] Donagi R., Witten E., Supersymmetric Yang-Mills theory and integrable systems, Nuclear Phys. B 460 (1996), 299-334, hep-th/9510101.

[5] Kapustin A., Sethi S., The Higgs branch of impurity theories, Adv. Theor. Math. Phys. 2 (1998), 571-591, hep-th/9804027.

[6] Seiberg N., Witten E., Gauge dynamics and compactification to three dimensions, hep-th/9607163.

[7] Klemm A., Lerche W., Mayr P., Vafa C., Warner N.P., Self-dual strings and $N=2$ supersymmetric field theory, Nuclear Phys. B 477 (1996), 746-766, hep-th/9604034.

[8] Witten E., Solutions of four-dimensional field theories via M-theory, Nuclear Phys. B 500 (1997) 3-42, hep-th/9703166.

[9] Chalmers G., Hanany A., Three dimensional gauge theories and monopoles, Nuclear Phys. B 489 (1997), 223-244, hep-th/9608105.

[10] Hanany A., Witten E., Type IIB superstrings, BPS monopoles, and three-dimensional gauge dynamics, Nuclear Phys. B 492 (1997), 152-190, hep-th/9611230.

[11] Katz S.H., Klemm A., Vafa C., Geometric engineering of quantum field theories, Nuclear Phys. B 497 (1997), 173-195, hep-th/9609239.

[12] Hitchin N., Brackets, forms and invariant functionals, math.DG/0508618.

[13] Kapustin A., Witten E., Electric-magnetic duality and the geometric Langlands program, hep-th/0604151.

[14] Hanany A., Tong D., On monopoles and domain walls, Comm. Math. Phys. 266 (2006), 647-663, hep-th/0507140. 
[15] Gerasimov A.A., Shatashvili S.L., Higgs bundles, gauge theories and quantum groups, hep-th/0609024.

[16] Donaldson S.K., Kronheimer P.B., The geomerty of four-manifolds, Oxford University Press, 1990.

[17] Jardim M., A survey on Nahm transform, J. Geom. Phys. 52 (2004), 313-327, math.DG/0309305.

[18] Nahm W., Self-dual monopoles and calorons, presented at 12th Colloq. on Group Theoretical Methods in Physics (September 5-10, 1983, Trieste, Italy).

Nahm W., Self-dual monopoles and calorons, Physics, Vol. 201, Springer, New York, 1984.

[19] Nahm W., All selfdual multi-monopoles for arbitrary gauge groups, presented at Int. Summer Inst. on Theoretical Physics (August 31 - September 11, 1981, Freiburg, West Germany).

[20] Jardim M., Construction of doubly-periodic instantons, Comm. Math. Phys. 216 (2001), 1-15, math.DG/9909069.

[21] Seiberg N., Witten E., Electric-magnetic duality, monopole condensation, and confinement in $N=2$ supersymmetric Yang-Mills theory, Nuclear Phys. B 426 (1994), 19-52, Erratum, Nuclear Phys. B 430 (1994), 485-486, hep-th/9407087.

[22] Seiberg N., Witten E., Monopoles, duality and chiral symmetry breaking in $N=2$ supersymmetric QCD, Nuclear Phys. B 431 (1994), 484-550, hep-th/9408099.

[23] Atiyah M.F., Hitchin N., The geometry and dynamics of magnetic monopoles, Princeton University Press, 1988.

[24] Diaconescu D.E., D-branes, monopoles and Nahm equations, Nuclear Phys. B 503 (1997), 220-238, hep-th/9608163.

[25] Cherkis S.A., Kapustin A., Singular monopoles and supersymmetric gauge theories in three dimensions, Nuclear Phys. B 525 (1998), 215-234, hep-th/9711145.

[26] Cherkis S.A., Kapustin A., Singular monopoles and gravitational instantons, Comm. Math. Phys. 203 (1999), 713-728, hep-th/9803160.

[27] Cherkis S.A., Hitchin N.J., Gravitational instantons of type D(k), Comm. Math. Phys. 260 (2005), 299-317, hep-th/0310084.

[28] Cherkis S.A., Kapustin A., Periodic monopoles with singularities and $N=2$ super-QCD, Comm. Math. Phys. 234 (2003), 1-35, hep-th/0011081.

[29] Cherkis S.A., Kapustin A., Nahm transform for periodic monopoles and $N=2$ super Yang-Mills theory, Comm. Math. Phys. 218 (2001), 333-371, hep-th/0006050.

[30] Howe P.S., Lambert N.D., West P.C., Classical M-fivebrane dynamics and quantum $N=2$ Yang-Mills, Phys. Lett. B 418 (1998), 85-90, hep-th/9710034.

[31] Hitchin N.J., Monopoles and geodesics, Comm. Math. Phys. 83 (1982), 579-602.

[32] Hitchin N.J., On the construction of monopoles, Comm. Math. Phys. 89 (1983), 145-190.

[33] Jardim M., Nahm transform and spectral curves for doubly-periodic instantons, Comm. Math. Phys. 225 (2002) 639-668.

[34] Donaldson S.K., Nahm's equations and the classification of monopoles, Comm. Math. Phys. 96 (1984), 387-407.

[35] Friedman R., Morgan J., Witten E., Vector bundles and F theory, Comm. Math. Phys. 187 (1997), 679-743, hep-th/9701162.

[36] Friedman R., Morgan J.W., Witten E., Vector bundles over elliptic fibrations, alg-geom/9709029.

[37] Simpson C.T., Higgs bundles and local systems, IHES Publ. Math. 75 (1992), 5-95.

Simpson C.T., The Hodge filtration on nonabelian cohomology, in Algebraic Geometry (1995, Santa Cruz), Proc. Symp. Pure Math., Vol. 62, Part 2, Amer. Math. Soc., Providence, 1997, 217-281, alg-geom/9604005. 